\title{
Viscoelastic properties of waxy crude oil
}

\author{
ZHANG Fan(张 帆) $)^{1,2}$, CHEN Guo-qun(陈国群) ${ }^{1}$ \\ (1. Beijing Key Laboratory of Urban Oil and Gas Distribution Technology, \\ China University of Petroleum (Beijing), Beijing 102249, China; \\ 2. Institute of Mechanics, Chinese Academy of Sciences, Beijing 100081, China)
}

\begin{abstract}
Waxy crude oil usually represents viscoelastic property when temperature is below its wax appearance temperature (WAT). The more the waxy crystals precipitated in crude oil, the stronger the structure and the viscoelasticity formed by waxy crystals. As the structure of waxy crystals relates to viscoelasticity that can be described by the storage modulus $G^{\prime}$ and loss modulus $G^{\prime \prime}$, the small amplitude oscillation (SAOS) method was introduced to determine these moduli. This shows that the temperature at which storage modulus equals loss modulus is just the pour point of waxy crude oil. The structure intensity or yield stress of waxy crude oil can be determined by $\tau_{y}^{\prime}=G^{\prime} / 50$. In this way wall slid can be avoided. It also shows that 60 min. will at least be taken for waxy crystals to build up the structure completely in oil. It means that waxy crude oil sample should be stayed still at test temperature in the sensor of rheometer more than $60 \mathrm{~min}$. before the viscoelasticity or yield stress of waxy crude is tested. That is structure building time.
\end{abstract}

Key words: waxy crude; viscoelasticity; structure strength; small amplitude oscillation(SAOS)

\section{Introduction}

Waxy crude oil has high pour point and has to be pipelined by heating with the heating stations along the pipeline. After leaving heating station, the hot oil is gradually cooled during it flows in the pipeline. When temperature below its wax appearance temperature(WAT), waxy crystals will be crystallized gradually. And the lower the temperature is below WAT, the more the waxy crystals appear in oil. The waxy crystals connect each other to form net structure and make waxy crude oil show some non-Newtonian rheological properties, such as thixotropy, yield stress and viscoelasticity. If the structure of waxy crystals is strong enough, especially in the case of the pipeline shutdown, the waxy crude oil changes into gel and loses fluidness. Obviously, how to know waxy crystals structure and its strength is very important for waxy crude oil pipelining.

\section{Viscoelasticity measurement}

For viscoelasticity measurement, three methods, CR mode(controlled rate), CS mode(controlled stress) and SAOS mode(small amplitude oscillatory) are often used. Among them, SAOS mode is a good choice for viscoelastic measurement. As the amplitude of socillation is small enough, the relationship between shear stress and deformation is linear. In linear range the viscoelastic parameters of waxy crude have nothing to do with the stress applied on, they are only dependent upon the temperature of waxy crude. And waxy crystal structure is not disturbed or destroyed by the shear stress in linear range. When the measurement has been finished at one temperature, the next measurement at another temperature can be made directly. There is no need to replace the sample that has been used. Which makes it very convenient for tester to measure viscoelasticity at different temperature. It is also very helpful for tester to know the structure forming process by continuously observing the changes of viscoelasticity of waxy crude at same temperature and at different time.

In SAOS mode, the sine type shear stress $\tau$ with frequency $f$ is applied to the sample.

$$
\tau=\tau_{0} \sin (\omega t)
$$

where $\tau_{0}$ is the oscillation amplitude; $\omega=2 \pi f$ is the angular velocity.

The reactions of the sample are deformation $\gamma$ and phase displacement angle $\delta$ :

$$
\gamma=\gamma_{0} \sin (\omega t-\delta)
$$

Complex modulus $G^{*}$ is defined as $G^{*}=\tau_{0} / \gamma_{0}$, which represents the total resistance of the sample; Storage modulus $G^{\prime}$ is defined as $G^{\prime}=\tau_{0} / \gamma_{0} \cos \delta$, meaning elastic behavior of structure. Loss modulus $G^{\prime \prime}$ is defined as $G^{\prime \prime}=\tau_{0} / \gamma_{0} \sin \delta$, meaning viscous behavior of structure. A phase displacement angle of $0^{\circ}$ means elastic behavior and an angle of $90^{\circ}$ means 
viscous behavior while between them means viscoelastic behavior.

\subsection{Linear viscoelasticity range}

Linear range can be determined by stress scanning method with the rheometer that has the function of oscillation mode. For waxy crude oil, there are different linear ranges at different temperatures. The first thing before the viscoelasticity measured is to determine the linear range of waxy crude oil at different temperatures. For Zhongyuan waxy crude, one of the waxy crude samples of which pour point is about $36{ }^{\circ} \mathrm{C}$ and the result of stress scanning at $28^{\circ} \mathrm{C}$ and $1 \mathrm{~Hz}$ shows in Fig.1. The linear limit is about $15 \mathrm{~Pa}$ for Zhongyuan waxy crude.

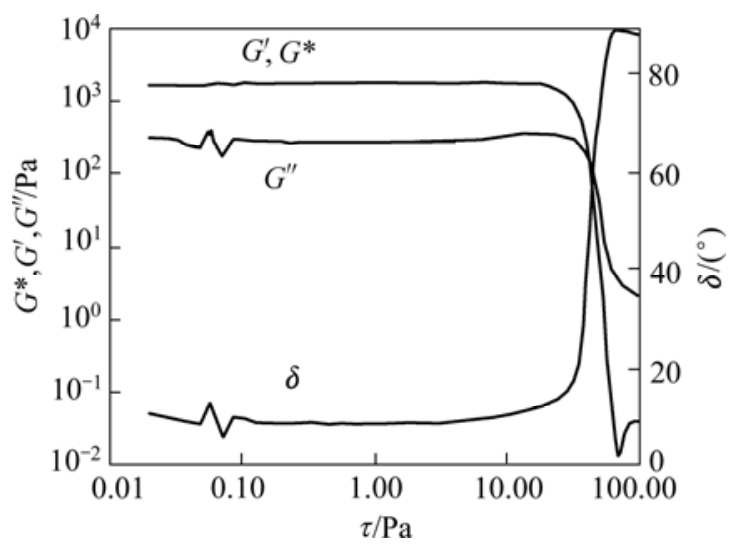

Fig.1 Linear range of Zhongyuan waxy crude

In this case, most of the waxy in the crude oil has crystalized and the structure formed by waxy crystals is very strong. So, $G^{\prime}$ is greater than $G^{\prime \prime},\left|G^{*}\right|=$ $\left(G^{\prime 2}+G^{\prime \prime \prime 2}\right)^{1 / 2}$ is very close to $G^{\prime \prime}$, which means that elastic behavior is dominant. Under the linear limit $15 \mathrm{~Pa}$, deformation $\gamma$ relates to shear stress $\tau$ linearly, no mater whatever the shear stress is, $G^{\prime}, G^{\prime \prime}$ and $\delta$ are almost the same. This means the structure of waxy crude oil is not destroyed. If the load is removed then the deformation will also disappear. When the shear stress is greater than $15 \mathrm{~Pa}$, with the stress increasing, $\delta$ increases sharply and $G^{\prime}$ decreases sharply. This means that the structure is broken. If the shear stress is great enough then $\delta=90^{\circ}$ in the end, it means the structure is broken totally and viscous behavior is dominant.

\subsection{Frequency of SAOS}

Although the storage modulus $G^{\prime}$ and loss modulus $G^{\prime \prime}$ are constant in the linear range of waxy crude oil, yet they are affected by frequence of sine type shear stress. Frequence $(f=2 \pi \omega)$ effect can be determined by frequency scanning test. The results of frequency scanning experiment, at $30{ }^{\circ} \mathrm{C}$, of Zhongyuan waxy crude are shown in Fig. 2 .

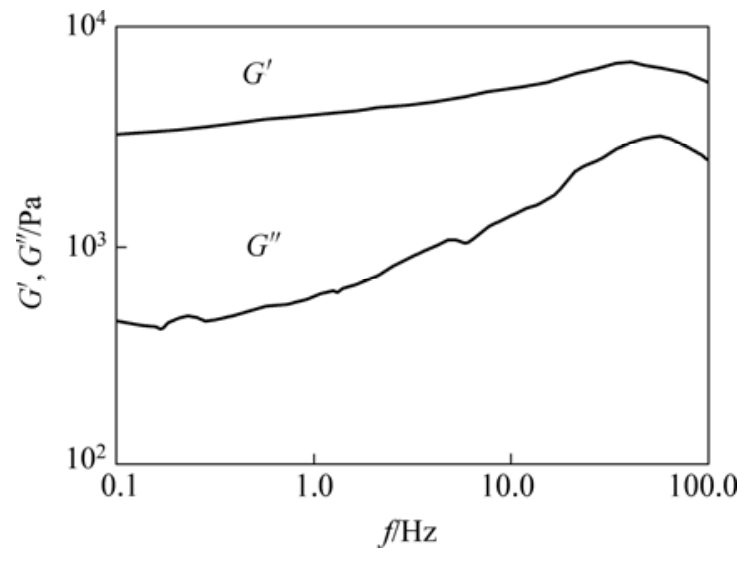

Fig.2 Frequence scanning of Zhongyuan waxy crude

It indicates that both $G^{\prime}$ and $G^{\prime \prime}$ are the functions of frequency. From low frequency to high frequency, they first increase then decrease with frequency increasing. That is, for $G^{\prime}$ and $G^{\prime \prime}$ there is a maximum value respectively at about $60 \mathrm{~Hz}$. As $G^{\prime}$ and $G^{\prime \prime}$ are the reactions of the sine type stress applied on the structure of waxy crude oil, unlike purely elastic body, the viscoelastic structure cannot respond instantly, at high frequency. Although low frequency is available, yet it will take a very long time to complete an experiment. As very high or very low frequency is not suitable for viscoelastic measurements, usually $1 \mathrm{~Hz}$ is selected in SAOS experiments.

\section{Viscoelasticity and pour point}

During the process that waxy crude oil changes from liquid state into gel state, its viscoelastic property gradually increases. In order to know viscoelasticity of waxy crude at different temperatures better, moduli of structure are tested in SAOS at different temperatures from high temperature to low temperature. It is also called temperature scanning experiment. The temperature decreasing rate should be very slow to assure that the temperature is homogeneous everywhere in the sample. The cooling rate less than $0.2{ }^{\circ} \mathrm{C} / \mathrm{min}$ is suggested.

Fig. 3 shows the results of the temperature scanning, less than $0.2{ }^{\circ} \mathrm{C} / \mathrm{min}$, at $1 \mathrm{~Hz}$, for Zhongyuan waxy crude oil.

It can be seen that beyond $35.5{ }^{\circ} \mathrm{C}, G^{\prime \prime}$ is always greater than $G^{\prime}$, that is, viscous property of waxy crude is stronger than elastic property. Below $35.5^{\circ} \mathrm{C}, G^{\prime}$ is less than $G^{\prime \prime}$. This means that the elastic property is stronger than viscous property. And the increasing rate of $G^{\prime}$ is greater than that of $G^{\prime \prime}$ with temperature decreasing. It indicates that the structure strength of waxy crystals gets 
stronger rapidly. At $35.5{ }^{\circ} \mathrm{C}$ two curves across, $G^{\prime}$ is equal to $G^{\prime \prime}$, and it is just the pour point temperature of Zhongyuan waxy crude.

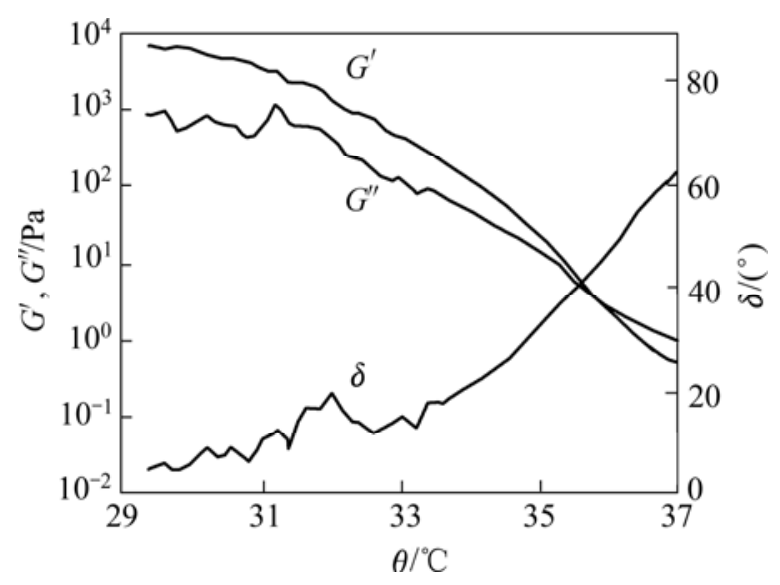

Fig.3 Temperature scanning of Zhongyuan waxy crude

The relationship between pour point temperature and the point of intersection of curve $G^{\prime}$ and curve $G^{\prime \prime}$ is also shown in other waxy crude oil in temperature scanning experiments in which the cooling rate is also less than $0.2^{\circ} \mathrm{C} / \mathrm{min}$ and the oscillation frequency is $1 \mathrm{~Hz}$ (see Table1).

Table 1 Pour point and cross point of waxy crude

\begin{tabular}{ccc}
\hline Waxy crude & Pour point $/{ }^{\circ} \mathrm{C}$ & Cross point $/{ }^{\circ} \mathrm{C}$ \\
\hline Zhongyuan & 36 & 35.5 \\
Daqing & 37 & 37 \\
Shengli & 20 & 20 \\
\hline
\end{tabular}

It can be seen from Table 1 that pour point and cross point agree each other very well. So pour point also can be measured by SAOS method under assigned conditions.

\section{Viscoelasticity and yield stress}

As mentioned above, when temperature below pour point, the network structure of waxy crystals makes crude oil be gel-like and lose fluidless. Only the structure is destroyed or its yield stress overcome, waxy crude can flow again. The lower the temperature below pour point, the stronger the network structure and the greater the yield stress of waxy crude. Yield stress stands for intensity of structure. So how to determine yield stress accurately is very important to know gelled waxy crude better. Usually with the rheometer, yield stress is measured by several methods involved concentriccylinder, parallel disk, and vane. The rotation rate and stress, torque and shear rate, and rotation under constant applied stress were measured respectively with each method $^{[1]}$. Yet every method has the same shortage that yield stress is not measured at zero shear rate and the wall slide problem is inevitable.

When the structure is broken by applied stress, the $G^{\prime}$ decreases sharply (see Fig.1). This means there must be some relationship between storage modulus $G^{\prime}$ and waxy crystals structure and its strength. The investigation has been done. As yield stress $\tau_{\mathrm{y}}$ can describe strength of network structure of waxy crystals either, for Zhongyuan waxy crude oil, $\tau_{\mathrm{y}}$ and $G^{\prime}$ were measured respectively by concentric cylinder method and SAOS method at different temperatures. The test results are shown in Fig.4. Comparing $\tau_{\mathrm{y}}$ with $G^{\prime} / 50$, they agree with each other very well at most testing temperatures, except $28{ }^{\circ} \mathrm{C}$, about $8{ }^{\circ} \mathrm{C}$ below pour point of Zhongyuan waxy crude oil. At this low temperature, almost all waxes in the oil have crystallized and the network structure of them is very strong. When yield stress of strong structure is tested by concentric-cylinder method, wall slide problem often occurs as the cylinder begins to move, which leads to that the tested result of yield stress is less than the real yield stress of waxy crude oil. Sometimes it is dangerous that the restart pressure of shutdown waxy crude oil pipeline is estimated by the yield stress involving wall slide.

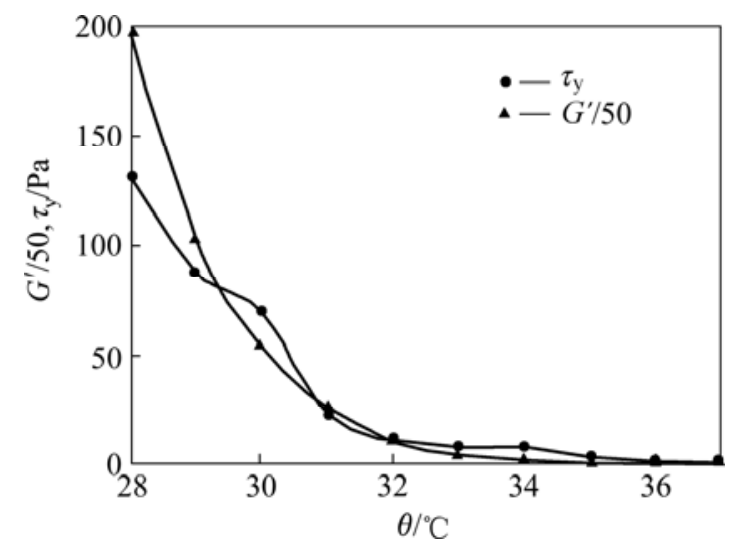

Fig.4 $G^{\prime} / 50$ and $\tau_{y}$ of Zhongyuan crude at different temperatures

But for SAOS method, there is no relative movement between the samples and testing sensor, so there is no wall slide at all. This leads to different results between two methods at low temperature. As is wall slide eliminated, $\tau_{\mathrm{y}}^{\prime}=G^{\prime} / 50$ can be used to describe structure strength of waxy crude oil more accurately. And without destroying waxy crystal structure, SAOS method need not change sample for different temperature measurements that should be done in traditional methods. And it is very efficient. 


\section{Structure building time}

While waxy crystals appear in oil, the structure of waxy crystals will build. It will take a period of time for building up structure entirely at testing temperature. As storage modulus $G^{\prime}$ relates to the structure, the process of structure building can be detected by detecting the changes of $G^{\prime}$ at the testing temperature. When temperature of the sample reaches the test temperature and is kept constant, the SAOS method is used to measure the $G^{\prime \prime}$ of the sample. The $G^{\prime \prime}$ of Daqing waxy crude oil at $28{ }^{\circ} \mathrm{C}$, about $8^{\circ} \mathrm{C}$ below its pour point, is measured (Fig.5).

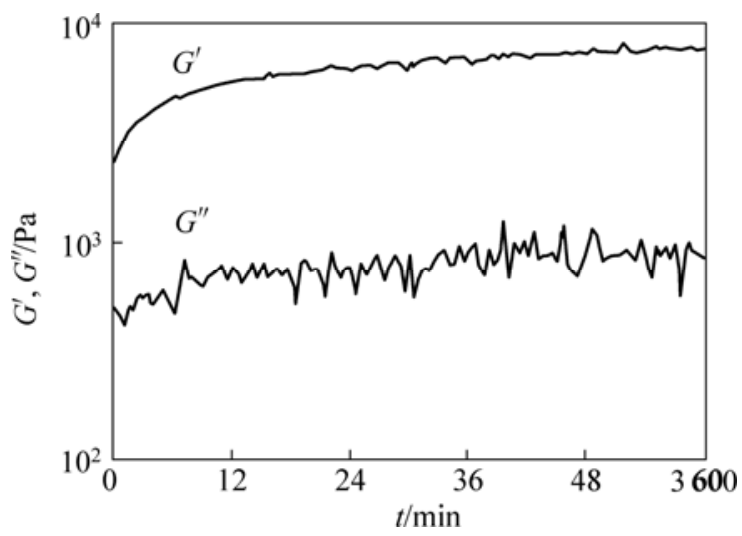

Fig.5 Daqing crude network building time

It can be seen from Fig. 5 that the storage modulus $G^{\prime}$ increases with the time increasing when the temperature is kept at the test temperature. It takes about 50 min for $G^{\prime}$ of Daqing waxy crude to close to its stable value. This means the structure is built up completely and its strength or yield stress reaches the maximum.

For different oil, it needs different time to build up the structure entirely. Fig. 6 shows building time for artificial waxy oil (white oil and wax). The test temperature is also lower $8{ }^{\circ} \mathrm{C}$ than its pour point. Its compositions are listed in Table 2.

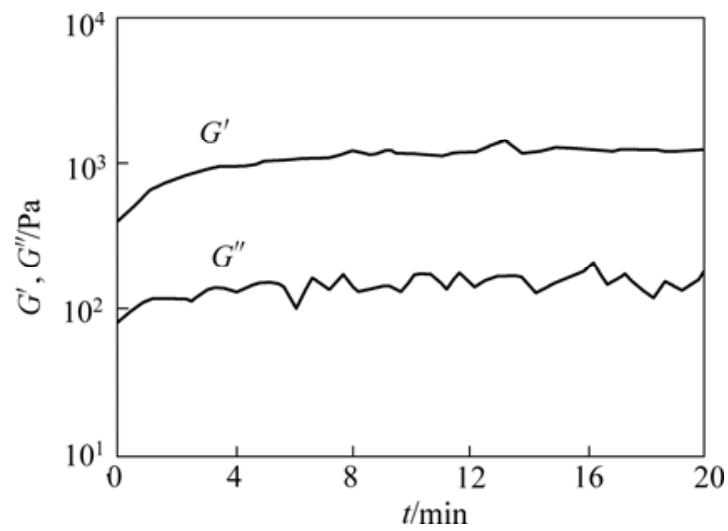

Fig.6 Artificial oil network building time
Table 2 Composition and building time

\begin{tabular}{ccccc}
\hline Sample & Wax $/ \%$ & Asphalt $\%$ & Resin/\% & $\begin{array}{c}\text { Building } \\
\text { time/min }\end{array}$ \\
\hline $\begin{array}{c}\text { Daqing } \\
\text { waxy crude }\end{array}$ & 21.51 & 10.19 & 0.17 & 50 \\
Artificial oil & 10 & 0 & 0 & 12 \\
\hline
\end{tabular}

It takes about $12 \mathrm{~min}$ for $G^{\prime}$ of artificial waxy oil to close to its stable value. Compared Daqing waxy crude oil with artificial waxy oil in composition and struction building time (see Table 2), it seems that not only the amount of wax in oil but also asphalt and resins may affect the structure building time. It is thought that asphalt and resins spread everywhere in the oil and they separate or insulate the waxy crystals to hinder the connection among waxy crystals. It may be the main cause to delay network building time of waxy crystals. So, in order to measure the yield stress or other properties correctly at low temperature(below pour point), no mater what measurement method and rheometer are used, about $60 \mathrm{~min}$ is suggested for the sample to stay at test temperature before the test is carried out.

\section{Conclusions}

1) Pour point can be measured with SAOS method by determining the point of intersection of curve $G^{\prime}$ and $G^{\prime \prime}$.

2) As the wall slide is avoided, the structure intensity or yield stress of waxy crude can be described by formula $\tau_{\mathrm{y}}^{\prime}=G^{\prime} / 50$ more accurately.

3) For waxy crude, 60min. is suggested for the sample to stay at desired temperature before test is made.

4) As without destroying waxy crystal structure, SAOS method need not change samples during the measurement at different temperatures. It is easy and efficient for the measurement.

\section{References}

[1] CAWKWELL M G, CHARLES M E. Characteristics of Canadian Arctic thixotropic gelled crude oil utilizing an eight-parameter model[J]. J Pipelines, 1989, 7: 251-264.

(Edited by LI Xiang-qun) 